\title{
Letter to the Editor: Frequency of the PD Allele
}

\author{
HAYATO KIHARA AND ARVAN L. FLUHARTY
}

UCLA, NPI-Pacific State Hospital Research Group, Pomona, California, USA

In our report on the prenatal diagnosis in a family with pseudo arylsulfatase A deficiency at risk for metachromatic leukodystrophy (MLD), we indicated that it was our impression that the frequency of the PD allele might be equal to or higher than the frequency of the MLD allele (1). The observations of Zlotogora et $a l$. and our recent involvement in five additional such prenatal diagnosis [one a repeat pregnancy in the index case (1)] have reinforced our view. A systematic study of the nuances of the present allelism requires identification of individuals with different genotypes. However, existing enzyme assay techniques do not allow differentiation between the MLD heterozygote and the PD heterozygote, and unequivocal differentiation between the PD homozygote and the MLD-PD mixed heterozygote is problematical. The approach taken by Zlotogora et al., namely studies of parents of known pseudodeficient subjects, will allow the use genetic considerations as well as biochemical information to ide tify individuals with different genotypes. In one family, genoty prediction was possible by genetic criteria alone. The positi identification of individuals with the various PD genotypes $\mathrm{w}$ greatly facilitate development of biochemical protocols applicat for population studies.

\section{REFERENCES AND NOTES}

1. Kihara, H., Ho, C. K., Fluharty, A. L., Tsay, K. K., and Hartlage, P. L.: Pren diagnosis of metachromatic leukodystrophy in a family with pseudo aryl fatase A deficiency by the cerebroside sulfate loading test. Pediatr. Res., 224 (1980). 